\title{
480Mbps / 1 Gbps radio-over-fiber link based on a directly modulated III-V-on-Silicon DFB laser
}

\author{
K. Van Gasse ${ }^{1}$, J. Van Kerrebrouck ${ }^{2}$, A. Abbasi ${ }^{1}$, G. Torfs ${ }^{2}$, H. Chen ${ }^{1}$, X. Yin $^{2}$, J. Bauwelinck ${ }^{2}$, G. Roelkens ${ }^{1}$ \\ ${ }^{1}$ Photonics Research Group, Ghent University/imec, Technologiepark-Zwijnaarde 15, B-9052 Ghent, Belgium \\ ${ }^{2}$ Design-IBCN Group, Dep. INTEC, Ghent University/iMinds /imec, Technologiepark-Zwijnaarde 15, B-9052 Ghent, Belgium
}

Kasper.vangasse@ugent.be

\begin{abstract}
In this work we characterize the RF modulation characteristics and the dynamic range of a III-V-on-Silicon DFB laser. The direct modulation of this laser is demonstrated with a 1Gbps radio-over-fiber link at a carrier frequency up to $24 \mathrm{GHz}$ using QPSK modulation. 480Mbps 64-QAM transmission at 3.5 GHz carrier frequency is demonstrated as well. In the 480 Mbps experiment both the transmitter and receiver are silicon photonic components, providing a SFDR of about $90 \mathrm{dBHz}^{2 / 3}$ for the complete optical link including receiver electronics as well.
\end{abstract}

Keywords-Silicon photonics; Optical fiber communication; Microwave photonics; Quantum well lasers.

\section{INTRODUCTION}

The goals for next generation mobile networks (5G) are very ambitious and achieving both spatial and spectral densification will be vital [1][2]. Spectral densification can happen by either opening up currently sparsely used bands, such as the $3.5 \mathrm{GHz}$ band or moving to higher frequencies such as the 24,28 or 60 $\mathrm{GHz}$ bands. Spatial densification can be realized by the use of cloud radio access network (C-RAN) architectures, where a central office services and coordinates several pico-cells. A key enabler for the deployment of pico-cells is the radio-overfiber link (RoF). RoF allows moving complex operations to generate and process the wireless signal to the central office, thereby keeping the cost of the remote antenna heads low.

Analogue links put a stringent requirement on the linearity of the used components. Therefore in most cases external modulation is preferred in radio over fiber links. However, directly modulated lasers have proven a viable alternative and have gained interest over the past years [3], given the compactness of the resulting solution. The realization of compact transceivers is of paramount importance for such CRAN architectures, considering the large number of pico-cells that needs to be addressed. Implementation of these transceivers on a silicon photonic platform is particularly attractive, as this integration platform allows the dense integration of high-speed optical functions at low cost. Recently a directly modulated III-V-on-silicon laser been demonstrated for digital links over several kilometers of fiber [4]. This type of laser can be co-integrated with passive optical functions such as wavelength multiplexing functionality and integrated high-speed photodetectors. The integration on silicon also enables close integration with electronics.

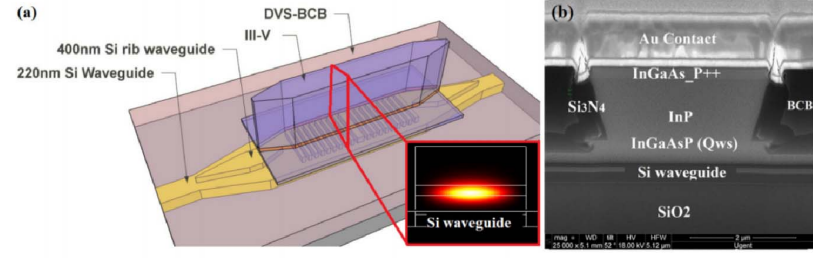

(c)

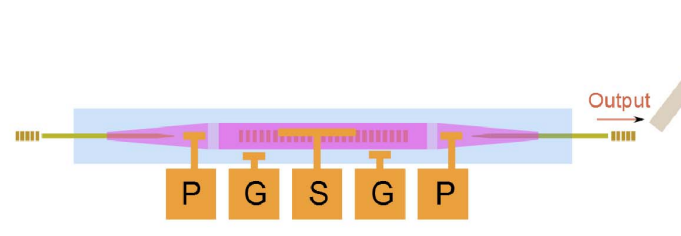

Figure 1: (a) A 3D representation of the III-V-on-Silicon DFB laser; (b) SEM cross-section of the laser; (c) Top view with schematic of the electrical and optical interface.

In the first part of this paper, a RoF link based on silicon photonic components will be demonstrated in the $3.5 \mathrm{GHz}$ band, as this band can be of interest in future networks both for distributed antenna systems as well as for small cell architectures. On the transmitter side a linear directly modulated III-V-on-Silicon DFB laser is used. On the receiver side a Germanium-on-silicon waveguide-coupled photodiode with co-integrated transimpedance amplifier is used [5]. 480 Mpbs data transmission, using a 64-QAM modulation scheme is demonstrated. Next, 1 Gbps data transmission is demonstrated at carrier frequencies of $3.5 \mathrm{GHz}, 20 \mathrm{GHz}$ and $24 \mathrm{GHz}$ using a QPSK modulation format. More complex modulation schemes considered for $5 \mathrm{G}$ applications will be tested in the near future.

The remainder of this work is structured as follows. In section II the RoF transmitter based on a III-V-on-silicon DFB laser is described in detail. In section III the $480 \mathrm{Mbps}$ RoF link based on this transmitter and a silicon photonic receiver is demonstrated. In section IV a $1 \mathrm{Gbps}$ RoF data link with the III-V-on-silicon laser transmitter is discussed. Finally section $\mathrm{V}$ summarizes the conclusions. 


\section{Silicon Photonic Transmitter}

A directly modulated III-V-on-silicon distributed feedback laser is used as transmitter in this work. This laser is manufactured on a silicon-on-insulator PIC containing passive waveguides, the distributed feedback grating and vertical fiber coupling gratings, as shown in Fig. 1. The gain section of the laser is integrated by adhesive die-to-wafer bonding of a multi-quantum well InP-based layer stack on the silicon PIC. This wafer bonding is achieved using a $10 \mathrm{~nm}$ thick polymer (DVS-BCB) layer. A more detailed description of the device fabrication can be found in [6]. In Fig. 2(a) the LI curve and IV curve of the laser are shown, at $20^{\circ} \mathrm{C}$. The laser is $400 \mu \mathrm{m}$ long. The threshold current of the laser is approximately 20 $\mathrm{mA}$ and the series resistance is $7 \Omega$. At an injection current of $100 \mathrm{~mA}$ the output of the laser reaches $3 \mathrm{~mW}$ at a wavelength of $1570 \mathrm{~nm}$. The laser output is single mode with a side mode suppression ratio of $40 \mathrm{~dB}$. The small signal modulation bandwidth of the laser was determined using a Keysight PNAX $67 \mathrm{GHz}$ network analyzer. The laser was contacted using a $40 \mathrm{GHz}$ bandwidth GSG RF probe. The $\mathrm{S}_{21}$ parameter for different injection currents is shown in Fig. 2(b). The bandwidth increases with increasing injection current due to a shift of the relaxation oscillation resonance. At an injection current of $100 \mathrm{~mA}$ the $3 \mathrm{~dB}$ bandwidth rises to $15 \mathrm{GHz}$, followed by a slow roll off. In this specific design the III-V-tosilicon tapers were electrically isolated from the laser section and can be pumped separately. This was found to improve the performance of the device.
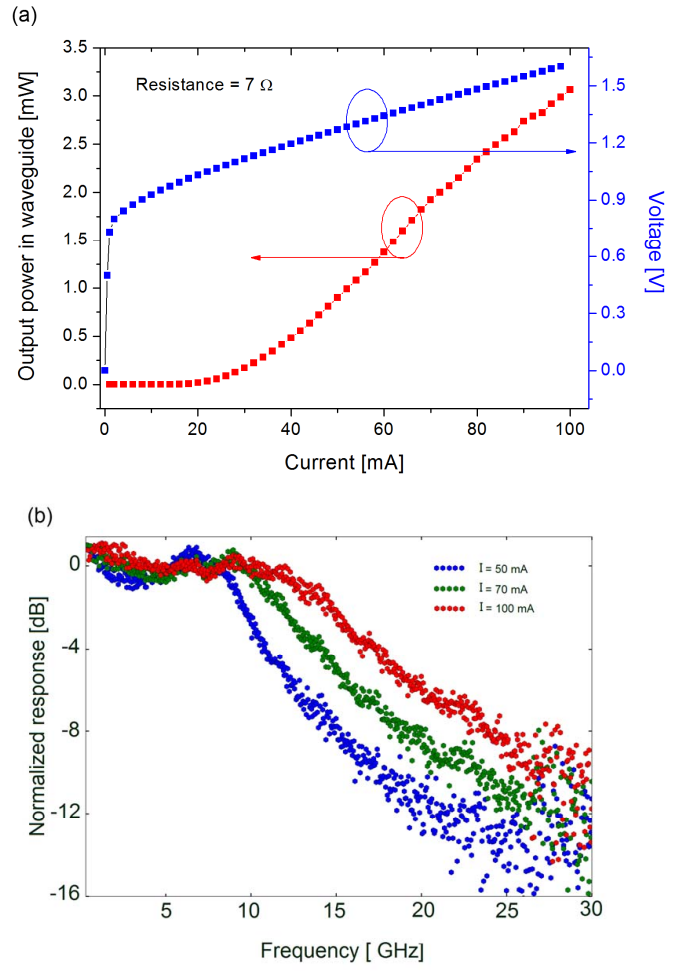

Figure 2: (a) III-V-on-silicon DFB laser LI and IV curve; (b) $\mathbf{S}_{21}$ small signal modulation transfer function.

\section{64-QAM 480 MBPS TRANSMISSION}

As a first demonstration a $5 \mathrm{~km}$ RoF link using a 64-QAM signal on a $3.5 \mathrm{GHz}$ carrier was realized. The layout of the setup is shown in Fig. 3. The III-V-on-silicon DFB was directly modulated using an Anritsu MS2692A signal generator. A SHF RF amplifier with $50 \mathrm{GHz}$ bandwidth was placed between the signal generator and DFB laser to increase the modulation depth of the laser as the on-chip laser was not impedance matched. The light from the on-chip laser was collected by a cleaved SMF fiber using a vertical grating coupler. The optical power in the fiber was $-3 \mathrm{dBm}$ and the fiber coupling losses are estimated at $7 \mathrm{~dB}$. The ouput was amplified with a Keopsys EDFA to $10 \mathrm{dBm}$ optical power, after which a Santec tunable optical filter (OTF-350) was used to filter out the ASE generated from the EDFA. An optical power of $6 \mathrm{dBm}$ was coupled into the receiver PIC again using a cleaved SMF and a vertical grating coupler. The optical power at the photodiode was estimated to be $-3 \mathrm{dBm}$ due to the grating coupler losses. A linear TIA [7] was integrated with the silicon receiver PIC using wire bonding and connected to an Anritsu signal analyzer used to demodulate the signal and determine the error vector magnitude (EVM).

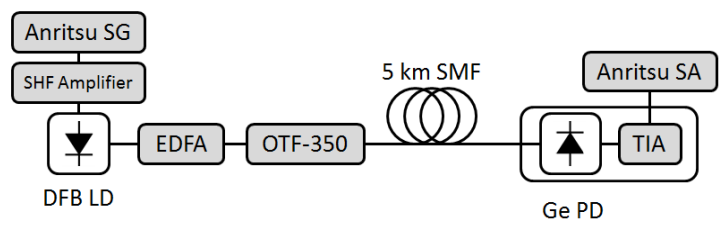

Figure 3 : Measurement set-up used for the 480 Mbps 64QAM RoF transmission.
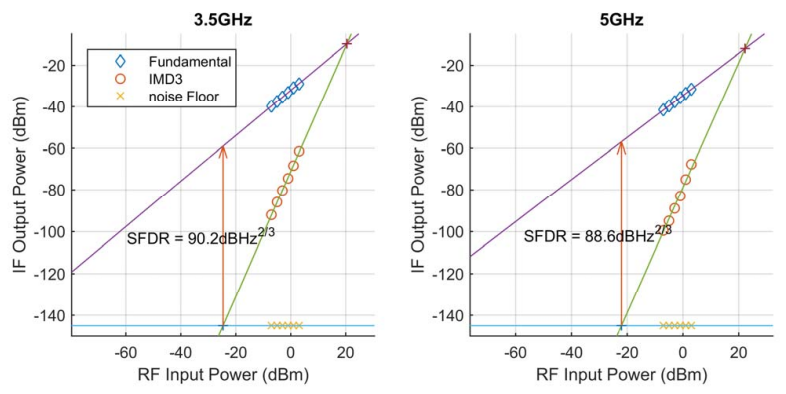

Figure 4: Dynamic range measurement of the entire optical link at a carrier frequency of $3.5 \mathrm{GHz}$ and $5 \mathrm{GHz}$.

A linearity measurement of the entire link is shown in Fig. 4. A SFDR of $90 \mathrm{dBHz}^{2 / 3}$, for a carrier frequency of $3.5 \mathrm{GHz}$ and a laser bias current of $100 \mathrm{~mA}$, is obtained. It was found that the linearity of the link depended strongly on the DC injection current of the DFB laser. This can be explained by the location of the relaxation oscillation resonance peak in the frequency response of the laser, which shifts to higher frequencies with increasing bias current. It is well-known that for the sake of linearity it is beneficial to work at a carrier frequency far away 
from this laser resonance frequency. We also measured the linearity at $5 \mathrm{GHz}$ and found a similar SFDR. The IIP3 of the link is approximately $20 \mathrm{dBm}$. Fig. 5(a) shows the constellation diagram of a $480 \mathrm{Mbps}$ 64-QAM signal transmitted over $5 \mathrm{~km}$ of single mode fiber. The measured rms error vector magnitude was 3.3 percent, showing good signal quality and promising performance.

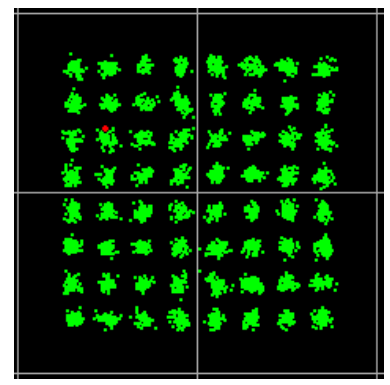

Figure 5: Constellation diagram of the 64 - QAM measurement.

\section{1GBPS QPSK TRANSMISSION AT 3.5 GHz, 20 GHz AND 24} GHZ CARRIER FREQUENCY

In a second demonstration a RoF link was set up using a 1 Gbps QPSK signal at different RF carrier frequencies. To achieve these higher data rates and higher carrier frequencies, an arbitrary waveform generator (AWG) and Keysight realtime DSOZ634A Oscilloscope with $63 \mathrm{GHz}$ bandwidth was used instead of the Anritsu signal generator and analyzer. The setup is shown in Figure 6.

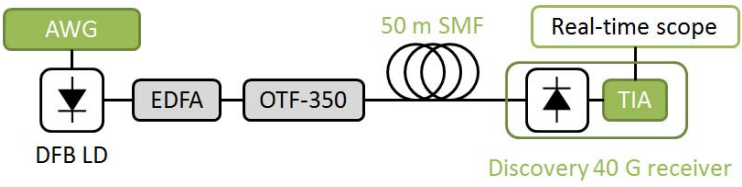

Figure 6: Measurement set-up of the $1 \mathrm{Gbps}$ RoF link.

The silicon photonic receiver was replaced by a Discovery photodiode with TIA, as the bandwidth of the available silicon photonic receiver was limited to $7 \mathrm{GHz}$. To focus on the signal quality as function of carrier frequency the $5 \mathrm{~km}$ single mode fiber was replaced with $50 \mathrm{~m}$ of single mode fiber. In a first experiment $1 \mathrm{Gbps}$ QPSK at a carrier frequency of $3.5 \mathrm{GHz}$ was transmitted. The constellation diagram and spectrum of the 1 Gbps QPSK signal can be seen in Fig. 7(top-left) and 7(top-right) respectively. The measurement showed an rms EVM of 3.4 percent, comparable to the 64-QAM constellation measurement of section III.

The carrier frequency was then increased to $20 \mathrm{GHz}$ on which again a 1 Gbps QPSK signal was modulated, while maintaining the same RF input power. The rms EVM degraded to 11.5 percent (see Fig. 7(bottom-left)), which is still very good for QPSK. However if we increase the carrier frequency to $24 \mathrm{GHz}$ the rms EVM degrades to 20.5 percent (see Fig. 7(bottom-right)). This can be expected as the laser is operated beyond its modulation bandwidth and the modulation efficiency decreases quickly. However, this rms EVM is still corresponding a BER below $10^{-5}$, which allows for forward error correction with limited overhead.
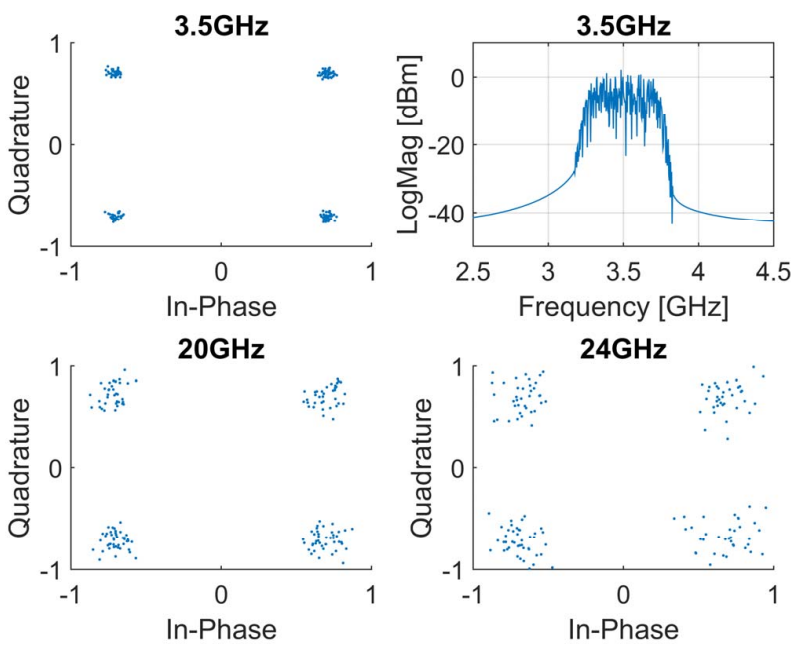

Figure 7: Top left: QPSK constellation plot of the received RoF signal at a carrier frequency of $3.5 \mathrm{GHz}$; Top right: spectrum at the signal analyzer; Bottom left: constellation diagram at $20 \mathrm{GHz}$ carrier frequency; Bottom right: constellation diagram at $24 \mathrm{GHz}$ carrier frequency.

\section{CONCLUSION}

For the first time, a RoF link based on a directly modulated III-V-on-Silicon DFB laser and a Germanium-on-Silicon waveguide-coupled photodiode is demonstrated. $480 \mathrm{Mbps}$ 64-QAM data transmission over $5 \mathrm{~km}$ single mode fiber on a $3.5 \mathrm{GHz}$ carrier is demonstrated. The use of silicon photonic components enables low-cost and densely integrated radioover-fiber transceivers, which will be essential for the realization of $5 \mathrm{G}$ cloud radio access networks (C-RANs) and distributed antenna systems. The possibility to achieve higher carrier frequencies and data rates is investigated as well, up to a carrier frequency of $24 \mathrm{GHz}$ and a data rate of 1 Gbps. While beyond $10 \mathrm{GHz}$ a commercial III-V receiver was used, recently silicon photonics based receivers with a small-signal bandwidth beyond $67 \mathrm{GHz}$ have been demonstrated [8], as well as III-V-on-silicon DFB lasers with a small-signal modulation bandwidth beyond $27 \mathrm{GHz}$ [9], making mm-wave radio-over-fiber transceivers/links based on silicon photonic components possible.

\section{AKNOWLEDGMENT}

This work was partly supported by the European Commission in the framework of the 2020-ICT-2014-2 project Flex5Gware (Grant agreement no. 671563), by the 
UGent special research fund (BOF14/GOA/034) and by the Hercules project AUGE/13/01.

\section{REFERENCES}

[1] N. Bhushan et al., "Network densification: the dominant theme for wireless evolution into 5G," in IEEE Communications Magazine, vol. 52, no. 2, pp. 82-89, February 2014.

[2] P. Rost et al., "Cloud technologies for flexible 5G radio access networks," in IEEE Communications Magazine, vol. 52, no. 5, pp. 68 76, May 2014.

[3] P. Hartmann, Xin Qian, A. Wonfor, R. V. Penty and I. H. White, "1-20 GHz Directly Modulated Radio over MMF Link," 2005 International Topical Meeting on Microwave Photonics, 2005, pp. 95-98.

[4] A. Abbasi, J. Verbist, J. V. Kerrebrouck, F. Lelarge, G. Duan, X. Yin, J. Bauwelinck, G. Roelkens, and G. Morthier, " $28 \mathrm{~Gb} / \mathrm{s}$ direct modulation heterogeneously integrated C-band InP/SOI DFB laser," Opt. Exp., vol. 23, no. 20, pp. 26479-26485, 2015.

[5] H. Chen, J. Verbist, P. Verheyen, P. De Heyn, G. Lepage, J. De Coster, P. Absil, X. Yin, J. Bauwelinck, J. Van Campenhout, G. Roelkens, "High sensitivity $10 \mathrm{~Gb} / \mathrm{s}$ Si photonic receiver based on a low-voltage waveguide-coupled Ge avalanche photodetector," Opt. Exp., vol. 23, no. 2, pp. 815-822, 2015.
[6] S. Keyvaninia, S. Verstuyft, L. Van Landschoot, F. Lelarge, G.-H. Duan, S. Messaoudene, J. M. Fedeli, T. De Vries, B. Smalbrugge, E. J. Geluk, J. Bolk, M. Smit, G. Morthier, D. Van Thourhout, and G. Roelkens, "Heterogeneously integrated III-V/silicon distributed feedback lasers," Opt. Lett., vol. 38, no. 24, pp. 5434-5437, 2013

[7] X. Yin, B. Moeneclaey, X. Qiu, J. Verbrugghe, K. Verheyen, J. Bauwelinck, J. Vandewege, M. Achouche, and Y. Chang, "A 10 Gb/s APD-based linear burst-mode receiver with $31 \mathrm{~dB}$ dynamic range for reach-extended PON systems", Opt. Exp.,vol. 20, no. 26, pp. 462-469, 2012.

[8] H. Chen, P. Verheyen, P. De Heyn, G. Lepage, J. De Coster, S. Balakrishnan, P. Absil, W. Yao, L. Shen, G. Roelkens, J. Van Campenhout, "-1 V bias $67 \mathrm{GHz}$ bandwidth Si-contacted germanium waveguide pin photodetector for 56Gbps optical links," Opt. Exp., vol. 24, no. 5, pp.4622-4631, 2016

[9] A. Abbasi, J. Verbist, X. Yin, F. Lelarge, G. H. Duan, J. Bauwelinck, G. Roelkens, G. Morthier, "Enhanced Modulation Bandwidth of Heterogeneously Integrated III-V-on-silicon DFB Laser for $40 \mathrm{~Gb} / \mathrm{s}$ NRZ-OOK Direct Modulation," International Semiconductor Laser Conference (ISLC), Japan, (to be published). 